\title{
Dynamic Sliding Mode Controller with Variable Structure for Fast Satellite Attitude Maneuver
}

\author{
Gao Shan, ${ }^{1}$ Li You $\mathbb{D}^{2},{ }^{2}$ Xue Huifeng, ${ }^{1}$ and Yao ShuYue ${ }^{2}$ \\ ${ }^{1}$ School of Automation, Northwestern Polytechnical University, Xi'an, China \\ ${ }^{2}$ School of Aerospace Science and Technology, Xidian University, Xi'an, China \\ Correspondence should be addressed to Li You; liyou@xidian.edu.cn
}

Received 25 January 2021; Revised 18 March 2021; Accepted 6 April 2021; Published 27 April 2021

Academic Editor: Xiaodong Sun

Copyright (c) 2021 Gao Shan et al. This is an open access article distributed under the Creative Commons Attribution License, which permits unrestricted use, distribution, and reproduction in any medium, provided the original work is properly cited.

In order to deal with the low convergence rate of the standard sliding mode in satellite attitude control, a novel variable structure sliding mode is constructed in this paper by designing the update law of the sliding mode parameter. By implementing this method, the advantage such as simple structure and strong robustness of the standard sliding mode are maintained and the system convergence rate is largely improved. The fixed sliding mode parameter is modified, and the update law is designed. When the system state is away from the sliding mode surface, the parameter is fixed, and when the system state approaches the sliding mode surface, the parameter begins to update. The constraint on control torque and angular velocity is taken into consideration, and the constraint on control parameters is given to ensure that the system state do not exceed its upper bound. System stability is proved by the Lyapunov stability theorem, and the superiority of the proposed controller is demonstrated by numerical simulation.

\section{Introduction}

In the satellite attitude control issue, sliding mode control has drawn much attention because of its simple structure and clear physical meaning. Researchers have done a lot of work focusing on sliding mode controllers for satellite attitude control. Xiao [1, 2] and $\mathrm{Hu}[3,4]$ have done some work for satellite attitude control, and some typical sliding modes are proposed in their work. The focus of their work is system robustness for model uncertainty, unknown disturbance, and actuator failure. $\mathrm{Wu}[5,6]$ also proposed some methods dealing with the chattering issue in sliding mode controllers. Most of these works mentioned above pointed out that strong robustness is an advantage of the standard sliding mode controller, but the exponential convergence rate (i.e., low convergence rate issue) near the system equilibrium point is its main drawback.

In order to improve the system convergence rate governed by sliding mode controllers, researchers developed some fast convergence control methods. Liang $[7,8]$ and Wang $[9,10]$ developed finite-time controllers for satellite attitude control. The focus of their work is the structure of the finite-time sliding mode, and some typical finite-time sliding modes are proposed. It is pointed out that the finite-time controller could largely improve the system convergence rate compared with the standard sliding mode but the cost is the complex structure of controllers which causes the loss of strong robustness. $\mathrm{Wu}$ [11] developed a finite-time controller based on the fast terminal sliding mode surface. The terminal sliding mode proposed by Dr. Wu in Ref [11] with finite time convergence rate is combined with standard sliding mode and a fractional order sliding mode. Hence, the system could have expected performance. $\mathrm{Li}$ [12] proposed a finite-time controller with a three-stage structure. A maneuver stage with constant angular velocity is constructed, and the angular velocity capability is fully utilized. Generally, finite-time control is an effective method to improve the system convergence rate but the cost is its complex structure and also the loss of strong robustness.

In order to have both fast convergence rate and strong robustness, researchers have done some modifications on the standard sliding mode. Jovan [13] proposed a simple structure sliding mode controller with the time varying parameter. System robustness is largely improved by the construction of the parameter update law. Although his 
main focus is on system robustness but not the convergence rate, the time varying sliding mode method proposed in his work is insightful. Verbin [14] and Lappas [15] also have done some work for satellite attitude control. They pointed that the key to improve the system convergence rate is to design the angular velocity trajectory properly. The braking curve for angular velocity is proposed in their work, and system performance is largely improved. $\mathrm{Li}$ [16-18] proposed some methods to design the angular velocity trajectory, and the system convergence rate is largely improved when compared with traditional methods. Sun [19-21] developed an improved direct instantaneous torque control based on the adaptive terminal sliding mode for a segmented-rotor SRM. A novel reaching law is proposed to reduce torque ripple and startup time. The modified sliding mode controllers are proposed, and system performance is largely improved. These works mentioned above could largely improve the system convergence rate, but the structure of the controller and sliding mode is still relatively complex and is hard for engineering practice.

Noting that the control capability is limited in engineering practice, excessive angular velocity would bring some unexpected characters (such as the failure of the star sensor, drop of attitude determination accuracy, large flexible deformation, and fuel consumption for reaction wheel damping); hence, it is necessary to consider control torque and angular velocity constraint when designing controllers. Hence, the goal of this paper could be concluded as follows: develop a sliding mode controller with simple structure and strong robustness under system control torque and angular velocity constraint. Also, the highlight and the main contribution of this paper could be concluded as follows: the standard sliding mode is modified by implementing a dynamic parameter update law; hence, the system convergence rate is improved, and this is a new method by the design sliding mode with expecting characters.

The structure of this paper could be listed as follows: the first part introduces the necessity of this paper, and this second part gives the dynamic and kinetic model used in this paper. The 3-5 parts are the main contributions of this paper, $3^{\text {rd }}$ part gives the controller, $4^{\text {th }}$ part discusses the constraint on control parameters, and $5^{\text {th }}$ part gives the numerical simulation and its analysis. The $6^{\text {th }}$ part concludes this paper.

\section{Dynamic and Kinetic Model}

The dynamic model of the satellite could be written as follows:

$$
J \dot{\omega}+\omega^{\times} J \omega=u+d,
$$

where $\omega$ is a three-dimensional vector which describes system angular velocity, $J$ is the inertia matrix of the satellite which is a $3 \times 3$ symmetric matrix, $d$ is three-dimensional unknown disturbance torque, and $u$ is a three-dimensional vector and the control torque generated by actuators such as thruster and reaction wheel, and the design of $u$ is the main focus of this paper. Product matrix $\mathbf{r}^{\times}$of vector $\mathbf{r}$ is defined as

$$
\mathbf{r}^{\times}=\left[\begin{array}{ccc}
0 & -r_{3} & r_{2} \\
r_{3} & 0 & -r_{1} \\
-r_{2} & r_{1} & 0
\end{array}\right] .
$$

Generally, inertia matrix $J$ could not be known accurately, and it could be described as follows:

$$
J=\widehat{J}+\widetilde{J}
$$

where $\widehat{J}$ is the inertia matrix best estimate and $\widetilde{J}$ is the error matrix.

Product matrix has an important property which will be used in the later part that the eigenvalues of $r^{\times}$satisfie

$$
\begin{aligned}
\lambda\left(\mathbf{r}^{\times}\right) & =0, \pm\|\mathbf{r}\|, \\
\lambda\left(\mathbf{m a x}^{\times}\right) & =\|\mathbf{r}\| .
\end{aligned}
$$

The kinetic model based on attitude quaternion could be written as follows:

$$
\begin{aligned}
& q=\left[\begin{array}{ll}
q_{0} & q_{v}^{T}
\end{array}\right]^{T}=\left[\cos \frac{\varphi}{2} e^{T} \sin \frac{\varphi}{2}\right]^{T}, \quad q_{0}=\cos \frac{\varphi}{2}, q_{v}=e \sin \frac{\varphi}{2}, \\
& \left\{\begin{array}{l}
\dot{q}_{0}=-\frac{1}{2} q_{v}^{T} \omega, \\
\dot{q}_{v}=\frac{1}{2}\left(q_{0} I_{3}+q_{v}^{\times}\right) \omega=\frac{1}{2} F \omega,
\end{array}\right.
\end{aligned}
$$

where $q_{0}$ and $q_{v}$ are the scalar part and vector part of attitude quaternion, respectively, with $\left|q_{0}\right| \leq 1, \quad q_{v}^{T} q_{v}+q_{0}^{2}=1$ and $\mathbf{e}$ and $\varphi$ are the Euler axis and Euler angle, respectively, and the definition is given in [12], and the eigenvalue of $F$ satisfies

$$
\begin{aligned}
\lambda(F) & =\left|q_{0}\right|, 1, \\
\lambda_{\max }(F) & =1 .
\end{aligned}
$$

In order to simplify the text, the maximum and minimum eigenvalue of matrix $A$ is described as $\lambda_{M}(A)$ and $\lambda_{m}(A)$.

\section{Variable Structure Sliding Mode Controller}

In the satellite attitude control field, the standard sliding mode surface could be written as follows:

$$
s=\omega+k q_{v}, \quad k>0 .
$$

When the system state reaches this sliding mode surface, we have

$$
\omega=-k q_{v}
$$

and

$$
\dot{q}_{0}=-\frac{1}{2} \omega^{T} q_{v}=\frac{1}{2} k q_{v}^{T} q_{v}=\frac{1}{2} k\left(1-q_{0}^{2}\right) .
$$

Based on equation (9), it is obvious that the system convergence rate is exponential. It is worth noticing that the reverse of angular velocity and attitude quaternion is an important property of this sliding mode surface. This 
property implies that when the norm of angular velocity is fixed, this sliding mode surface has the best efficiency on angular velocity, and because of this property, the standard sliding mode has been developed by researchers.

However, the main drawback of the standard sliding mode surface is its low convergence rate when the system state approaches its equilibrium point. The sliding mode parameter $k$ is fixed, and the norm of angular velocity is proportional to attitude quaternion, and when approaching the equilibrium point, the norm of angular velocity descends drastically, and this caused the low convergence rate of the standard sliding mode. If better convergence rate is needed, a common method is to enlarge the parameter, but this method would cause some unexpected characters such as severe vibration, large control torque, and angular velocity.

In order to solve the low convergence rate issue and avoid those drawbacks mentioned above, a variable structure sliding mode is proposed in this paper as follows:

$$
\begin{aligned}
& s=\omega+k q_{v}, \quad k(0)>0, \\
& \dot{k}= \begin{cases}0, & \|s\| \geq \varepsilon, \\
\frac{1}{2} p q_{0}^{3}\left(1-q_{0}\right), & \|s\|<\varepsilon,\end{cases}
\end{aligned}
$$

$$
u= \begin{cases}-\rho_{1} k_{s} s+\omega^{\times} \widehat{J} \omega-\frac{1}{2} k \widehat{J} F \omega-l_{1} \operatorname{sgn}(s), & \|s\| \geq \varepsilon, \\ -\rho_{2} k_{s} s+\omega^{\times} \widehat{J} \omega-\frac{1}{2} k \widehat{J} F \omega-\frac{1}{2} p q_{0}^{3}\left(1-q_{0}\right) \widehat{J} q_{v}-l_{2} \operatorname{sgn}(s), & \|s\|<\varepsilon\end{cases}
$$

where

$$
\left\{\begin{array}{l}
l_{1}=\bar{d}+\lambda\left(\|\omega\|^{2}+\frac{1}{2} k\|\omega\|\right) \\
l_{2}=\bar{d}+\lambda\left(\|\omega\|^{2}+\frac{1}{2} k\|\omega\|+\frac{1}{2} p q_{0}^{3}\left(1-q_{0}\right)\left\|q_{v}\right\|\right) .
\end{array}\right.
$$

where $k(0)$ is the initial value of sliding mode parameter $k$ and is a positive scalar, $p$ is a positive scalar that needs to be designed properly, and $\varepsilon$ is a small positive scalar.

It is obvious that sliding mode surface (10) is constructed as two stages: when the system state is away from the sliding mode surface i.e., $\|s\| \geq \varepsilon$, the proposed sliding mode is totally same as the standard sliding mode surface, and the parameter is fixed; when the system state reaches the sliding mode surface, i.e., $\|s\|<\varepsilon$, the angular velocity has been reversed to attitude quaternion and the sliding mode parameter begins to update. Based on the update law, it could be found that its derivative is positive scalar, and this means that the system could maneuver along the sliding mode surface with a small initial parameter, and the sliding mode parameter is enlarged when the system state approaches its equilibrium point. By this method, the norm of angular velocity would not drop so drastically as of the standard sliding mode; hence, the system convergence rate would be improved. It is worth noticing that on sliding mode surface (10), the angular velocity is reversed to attitude quaternion, and this means the advantage of the sliding mode could be maintained. Also, it could be found that when the system converges to its equilibrium point, the derivative of sliding mode parameters also tends to zero; hence, update law (10) does not have the singularity issue.

The attitude controller based on sliding mode (10) could be written as follows:

$$
\begin{gathered}
\rho_{i}= \begin{cases}\frac{-k_{s} s^{T} r_{i}+\sqrt{\left(k_{s} s^{T} r_{i}\right)^{2}+k_{s}^{2}\|s\|^{2}\left(\bar{u}^{2}-\left\|r_{i}\right\|^{2}\right)}}{k_{s}^{2}\|s\|^{2}}, & \|u\| \geq \bar{u}, \\
1, & \|u\|<\bar{u},\end{cases} \\
\begin{cases}r_{1}=\omega^{\times} \widehat{J} \omega-\frac{1}{2} k \widehat{J} F \omega-l_{1} \operatorname{sgn}(s), \\
r_{2}=\omega^{\times} \widehat{J} \omega-\frac{1}{2} k \widehat{J} F \omega-\frac{1}{2} p q_{0}^{3}\left(1-q_{0}\right) \widehat{J} q_{v}-l_{2} \operatorname{sgn}(s),\end{cases}
\end{gathered}
$$


where $\bar{u}$ is the norm upper bound of control torque. The goal of $\rho_{i}$ is to ensure that the control torque does not exceed its upper bound. Based on its definition, it could be found that when the demand torque does not exceed its upper bound, the factor does not change the controller structure, and when the demand torque exceeds its upper bound, $\rho_{i}$ could reduce the proportion term.

The next step is to prove system stability under controller (11). Select the Lyapunov function as follows:

$$
V_{s}=\frac{1}{2} s^{T} J s .
$$

When the system state does not reach the sliding mode, i.e., $\|s\| \geq \varepsilon$, calculate the derivative of (14) and substitute controller (11), and it could be obtained that

$$
\begin{aligned}
\dot{V}_{s} & =s^{T} J \dot{s}=s^{T} J \dot{\omega}+k s^{T} J \dot{q}_{v}, \\
& =s^{T}\left(u+d-\omega^{\times} J \omega\right)+\frac{1}{2} k s^{T} J F \omega, \\
& =-\rho_{1} k_{s} s^{T} s+s^{T} \omega^{\times} \widehat{J} \omega-\frac{1}{2} k s^{T} \widehat{J} F \omega-l_{1} s^{T} \operatorname{sgn}(s)+s^{T} d-s^{T} \omega^{\times} J \omega+\frac{1}{2} k s^{T} J F \omega, \\
& =-\rho_{1} k_{s} s^{T} s-s^{T} \omega^{\times} \widetilde{J} \omega+\frac{1}{2} k s^{T} \widetilde{J} F \omega+s^{T} d-\left(\bar{d}+\lambda\left(\|\omega\|^{2}+\frac{1}{2} k\|\omega\|\right)\right) s^{T} \operatorname{sgn}(s), \\
& \leq-\rho_{1} k_{s} s^{T} s \leq 0 .
\end{aligned}
$$

When the system state reaches the sliding mode, i.e., $\|\mathbf{s}\|<\varepsilon$, calculate the derivative of (14) and substitute controller (11), and it could be obtained that

$$
\begin{aligned}
\dot{V}_{s}= & s^{T} J \dot{s}=s^{T} J \dot{\omega}+k s^{T} J \dot{q}_{v}+\dot{k} s^{T} J q_{v}, \\
= & s^{T}\left(u+d-\omega^{\times} J \omega\right)+\frac{1}{2} k s^{T} J F \omega+\frac{1}{2} p q_{0}^{3}\left(1-q_{0}\right) s^{T} J q_{v}, \\
= & -\rho_{2} k_{s} s^{T} s+s^{T} \omega^{\times} \widehat{J} \omega-\frac{1}{2} k s^{T} \widehat{J} F \omega-\frac{1}{2} p q_{0}^{3}\left(1-q_{0}\right) s^{T} \widehat{J} q_{v}-l_{2} s^{T} \operatorname{sgn}(s) \\
& +s^{T} d-s^{T} \omega^{\times} J \omega+\frac{1}{2} k s^{T} J F \omega+\frac{1}{2} p q_{0}^{3}\left(1-q_{0}\right) s^{T} J q_{v}, \\
= & -\rho_{2} k_{s} s^{T} s+s^{T} d-s^{T} \omega^{\times} \widetilde{J} \omega+\frac{1}{2} k s^{T} \widetilde{J} F \omega+\frac{1}{2} p q_{0}^{3}\left(1-q_{0}\right) s^{T} \widetilde{J} q_{v} \\
& -\left(\bar{d}+\lambda\left(\|\omega\|^{2}+\frac{1}{2} k\|\omega\|+\frac{1}{2} p q_{0}^{3}\left(1-q_{0}\right)\left\|q_{v}\right\|\right)\right) s^{T} \operatorname{sgn}(s) \\
\leq & -\rho_{2} k_{s} s^{T} s \leq 0 .
\end{aligned}
$$

Hence, the sliding mode state $s$ is globally asymptotically stable, and based on the discussion above, systems (1) and (5) are globally asymptotically stable on sliding mode surface (10); hence, systems (1) and (5) governed by controller (11) are globally asymptotically stable. System stability has been proved.

\section{System State Constraint}

Generally, in order to get better convergence rate, the initial value of sliding mode parameter $k(0)$ and parameter $p$ should be selected as large as possible. However, it is obvious that larger control parameter would cause larger control 
torque and angular velocity, and system performance would be harmed, and the system state would diverge under some extreme cases. Hence, it is necessary to discuss the relation between angular velocity upper bound $\bar{\omega}$, control torque upper bound $\bar{u}$, and control parameters.

Firstly, the constraint of control torque $\bar{u}$ on control parameters is given. Noting that when the system state does not reach the sliding mode, the proportion term $k_{s} s$ could be reduced by the gain factor $\rho_{i}$; hence, the first term of controller (11) does not have the control torque saturation issue, and the key to satisfy the control torque constraint is the later terms. Noting that when $\|s\| \geq \varepsilon$,

$$
\begin{aligned}
\left\|r_{1}\right\| & =\left\|\omega^{\times} \widehat{J} \omega-\frac{1}{2} k \widehat{J} F \omega-l_{1} \operatorname{sgn}(s)\right\|, \\
& \approx\left\|\omega^{\times} J \omega+\frac{1}{2} k J F \omega\right\|, \\
& \leq \lambda_{M}(J)\left(\bar{\omega}^{2}+\frac{1}{2} k(0) \bar{\omega}\right),
\end{aligned}
$$

where $\bar{\omega}$ is the norm upper bound of angular velocity; hence, if the following inequality is satisfied, the control torque constraint could be satisfied:

$$
\lambda_{M}(J)\left(\bar{\omega}^{2}+\frac{1}{2} k(0) \bar{\omega}\right) \leq \bar{u} .
$$

When the system state reaches the sliding mode surface, the angular velocity satisfies

$$
\omega=-k q_{v} .
$$

Noting that

$$
\begin{aligned}
q_{0}^{3}\left(1-q_{0}\right) & =\frac{1}{3} q_{0}^{3}\left(3-3 q_{0}\right) \leq \frac{1}{3}\left(\frac{q_{0}+q_{0}+q_{0}+3-3 q_{0}}{4}\right)^{4} \\
& =\frac{27}{256}<\frac{1}{9} .
\end{aligned}
$$

Hence,

$$
\begin{aligned}
\left\|r_{2}\right\| & =\left\|\omega^{\times} \widehat{J} \omega-\frac{1}{2} k \widehat{J} F \omega-\frac{1}{2} p q_{0}^{3}\left(1-q_{0}\right) \widehat{J} q_{v}-l_{2} \operatorname{sgn}(s)\right\|, \\
& \approx\left\|\omega^{\times} J \omega-\frac{1}{2} k J F \omega-\frac{1}{2} p q_{0}^{3}\left(1-q_{0}\right) J q_{v}\right\|, \\
& =\left\|k^{2} q_{v}^{\times} J q_{v}+\frac{1}{2} k^{2} q_{0} J q_{v}-\frac{1}{2} p q_{0}^{3}\left(1-q_{0}\right) J q_{v}\right\|, \\
& \leq \lambda_{M}(J)\left(k^{2}\left\|q_{v}\right\|^{2}+\left(\frac{1}{2} k^{2}+\frac{1}{18} p\right)\left\|q_{v}\right\|\right), \\
& \leq \lambda_{M}(J)\left(\frac{3}{2} k^{2}+\frac{1}{18} p\right)\left\|q_{v}\right\| .
\end{aligned}
$$

Define auxiliary variable $z$ as follows:

$$
z=\lambda_{M}(J)\left(\frac{3}{2} k^{2}+\frac{1}{18} p\right)\left\|q_{v}\right\| .
$$

It is obvious that $z$ is the norm upper bound of $r_{2}$; hence, if $z \leq \bar{u}$ is satisfied, $\left\|r_{2}\right\| \leq \bar{u}$ could be ensured. Calculate the derivative of (22) and it could be obtained that

$$
\begin{aligned}
\dot{z} & =\lambda_{M}(J)\left(3 k \dot{k}\left\|q_{v}\right\|+\left(\frac{3}{2} k^{2}+\frac{1}{18} p\right) \frac{d\left\|q_{v}\right\|}{d t}\right), \\
& =\frac{1}{2} \lambda_{M}(J) k q_{0}\left\|q_{v}\right\|\left(3 p q_{0}^{2}\left(1-q_{0}\right)-\frac{3}{2} k^{2}-\frac{1}{18} p\right) .
\end{aligned}
$$

Hence, if $z$ reaches its maximum value, the following inequality holds:

$$
k^{2}=2 p q_{0}^{2}\left(1-q_{0}\right)-\frac{1}{27} p .
$$

Hence,

$$
\begin{aligned}
z & \leq 3 \lambda_{M}(J) p q_{0}^{2}\left(1-q_{0}\right)\left\|q_{v}\right\|=3 \lambda_{M}(J) p q_{0}^{2}\left(1-q_{0}\right) \sqrt{1-q_{0}^{2}}, \\
& =3 \lambda_{M}(J) p \sqrt{q_{0}^{4}\left(1-q_{0}\right)^{2}\left(1-q_{0}^{2}\right)}, \\
& \leq 3 \sqrt{2} \lambda_{M}(J) p \sqrt{q_{0}^{3}\left(1-q_{0}\right)^{3},} \\
& \leq \frac{3 \sqrt{2}}{8} \lambda_{M}(J) p .
\end{aligned}
$$

Since the following inequality holds, the control torque constraint could be ensured:

$$
\frac{3 \sqrt{2}}{8} \lambda_{M}(J) p \leq \bar{u} .
$$

Above all, the constraint of control torque on control parameters could be concluded as follows:

$$
\left\{\begin{array}{l}
\lambda_{M}(J)\left(\bar{\omega}^{2}+\frac{1}{2} k(0) \bar{\omega}\right) \leq \bar{u}, \\
\frac{3 \sqrt{2}}{8} \lambda_{M}(J) p \leq \bar{u} .
\end{array}\right.
$$

The former inequality is the constraint on the initial value of the sliding mode parameter, and the later inequality is the constraint on the update parameter. If they both are satisfied, system control torque would not exceed its norm upper bound during the whole control process.

The next step is to discuss the constraint of angular velocity on control parameters. Noting that, before reaching the sliding mode surface, the sliding mode structure is totally same as the standard sliding mode, and the method on the standard sliding mode could be implemented. Noting that, under this condition system could be treated as a two-stage linear system, and the overshot of the system state generally is usually less than $100 \%$; hence, by selecting control parameters as follows, the constraint of angular velocity could be ensured: 


$$
k(0) \leq \frac{1}{2} \bar{\omega} .
$$

When it reaches the sliding mode surface, calculate the derivative of the angular velocity norm:

$$
\begin{aligned}
\frac{d\|\omega\|}{d t} & =\dot{k}\left\|q_{v}\right\|+k \frac{d\left\|q_{v}\right\|}{d t}, \\
& =\frac{1}{2} p q_{0}^{3}\left(1-q_{0}\right)\left\|q_{v}\right\|-\frac{1}{2} k^{2} q_{0}\left\|q_{v}\right\|, \\
& =\frac{1}{2} q_{0}\left\|q_{v}\right\|\left(p q_{0}^{2}\left(1-q_{0}\right)-k^{2}\right) .
\end{aligned}
$$
value,

When the norm of angular velocity reaches its maximum

$$
k^{2}=p q_{0}^{2}\left(1-q_{0}\right)
$$

Hence, we have

$$
\begin{aligned}
\|\omega\| & =k\left\|q_{v}\right\| \leq \sqrt{p q_{0}^{2}\left(1-q_{0}\right)} \sqrt{1-q_{0}^{2}} \\
& \leq \sqrt{2 p} \sqrt{q_{0}^{2}\left(1-q_{0}\right)^{2}} \leq \frac{\sqrt{2 p}}{4} .
\end{aligned}
$$

Hence, the constraint of angular velocity on control parameters could be concluded as follows:

$$
\left\{\begin{array}{l}
k(0) \leq \frac{1}{2} \bar{\omega} \\
\frac{\sqrt{2 p}}{4} \leq \bar{\omega}
\end{array}\right.
$$

The former inequality is the constraint on the initial value of the sliding mode parameter, and the later inequality is the constraint on the update parameter. If they are both satisfied, system angular velocity would not exceed its norm upper bound during the whole control process.

\section{Numerical Simulation and Analysis}

Set system configurations as follows:

$$
\begin{aligned}
J & =\operatorname{diag}\left(\left[\begin{array}{lll}
30 & 25 & 20
\end{array}\right]\right) \mathrm{kg} \cdot \mathrm{m}^{2}, \widehat{J}=\operatorname{diag}\left(\left[\begin{array}{lll}
22 & 24 & 21
\end{array}\right]\right) \mathrm{kg} \cdot \mathrm{m}^{2}, \lambda=2, \\
t_{\text {sample }} & =0.5 s, \bar{d}=0.001 \mathrm{~N} \cdot m, \bar{u}=0.5 \mathrm{~N} \cdot m, \bar{\omega}=0.1 \frac{\mathrm{rad}}{\mathrm{s}}, \\
\omega\left(t_{0}\right) & =\left[\begin{array}{lll}
-0.03 & -0.04 & 0.05
\end{array}\right]^{T} \frac{\mathrm{rad}}{s}, q\left(t_{0}\right)=\left[\begin{array}{ll}
0 & \frac{\sqrt{6}}{6} \frac{\sqrt{3}}{3} \frac{\sqrt{2}}{2}
\end{array}\right]^{T} .
\end{aligned}
$$

In order to demonstrate the superiority of the proposed controller, the standard sliding mode controller is compared as follows:

$$
\begin{aligned}
& s=\omega+k q_{v}, \\
& u=-k_{s} s+\omega^{\times} J \omega-\frac{1}{2} k J F \omega-\bar{d} \operatorname{sgn}(s) .
\end{aligned}
$$

Case 1. Standard sliding mode controller with the large parameter.

Generally, larger control parameters in (34) could bring better a convergence rate. In order to select control parameters properly, the method to determine control parameters in [12] is referred in this paper, and following parameters are selected:

$$
k_{s}=2, k=0.25 \text {. }
$$

The simulation results are shown as follows:

Based on Figures 1 and 2, it could be found that system convergence time is about $90 \mathrm{~s}$; however, Figure 3 demonstrates that the initial control torque is relative large, and there exists a $10 \mathrm{~s}$ period with control torque exceeding the system norm upper bound. Also, based on Figure 3, it could be found that there exists a $15 \mathrm{~s}$ period with angular velocity exceeding the system upper bound. This group of simulation demonstrates that the standard sliding mode could have a good convergence rate by selecting the large parameter but the cost is excessive control torque and angular velocity.

Case 2. Standard sliding mode controller with the small parameter.

Based on the simulation results above, it could be found that system angular velocity exceeds its upper bound; hence, following parameters are selected:

$$
k_{s}=2, k=0.1
$$

The simulation results are shown as follows:

In this group of simulation, Figures 4 and 5 demonstrate that system convergence time is about $150 \mathrm{~s}$, and Figure 6 demonstrates that system control torque and angular velocity are both lower than the system upper bound. Comparing this group with Case 1 , it could be easily found that if control torque and angular velocity constraints are satisfied 


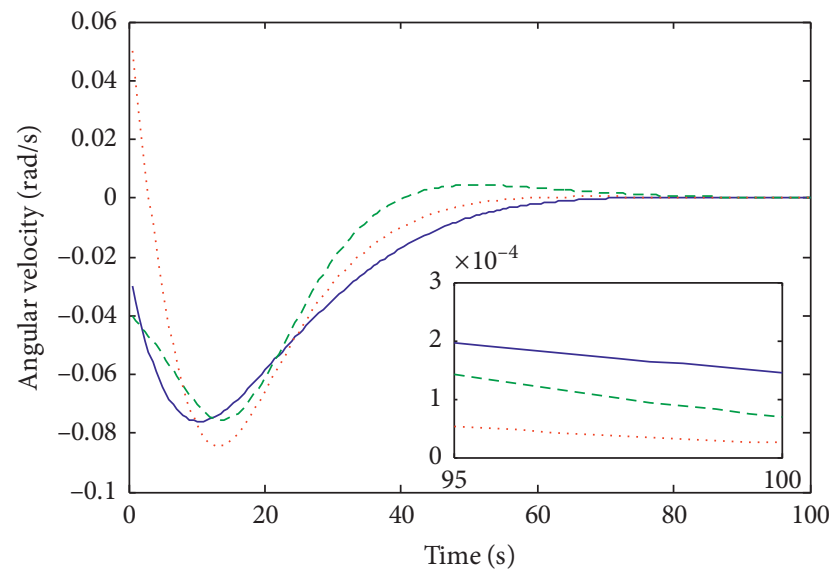

$-w x$

$---w y$

…w wz

FIgURE 1: Curve of angular velocity.

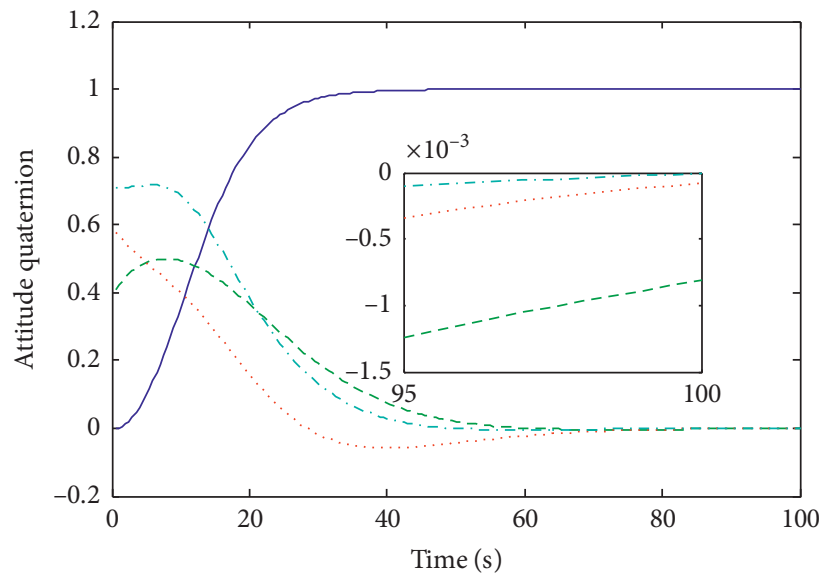

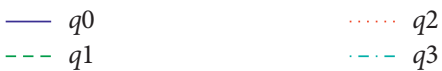

Figure 2: Curve of attitude quaternion.

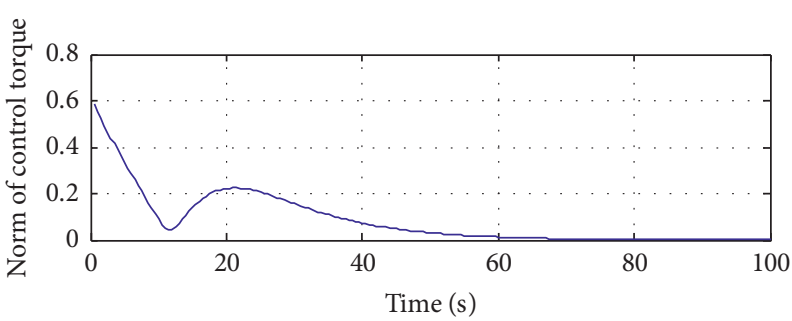

(a)

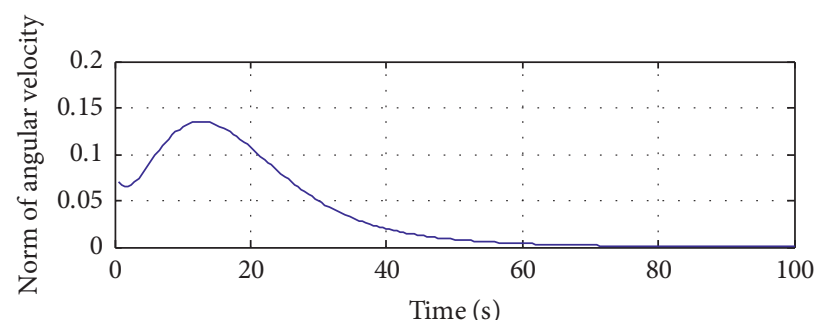

(b)

FIgURE 3: Curve of angular velocity and control torque norm. 


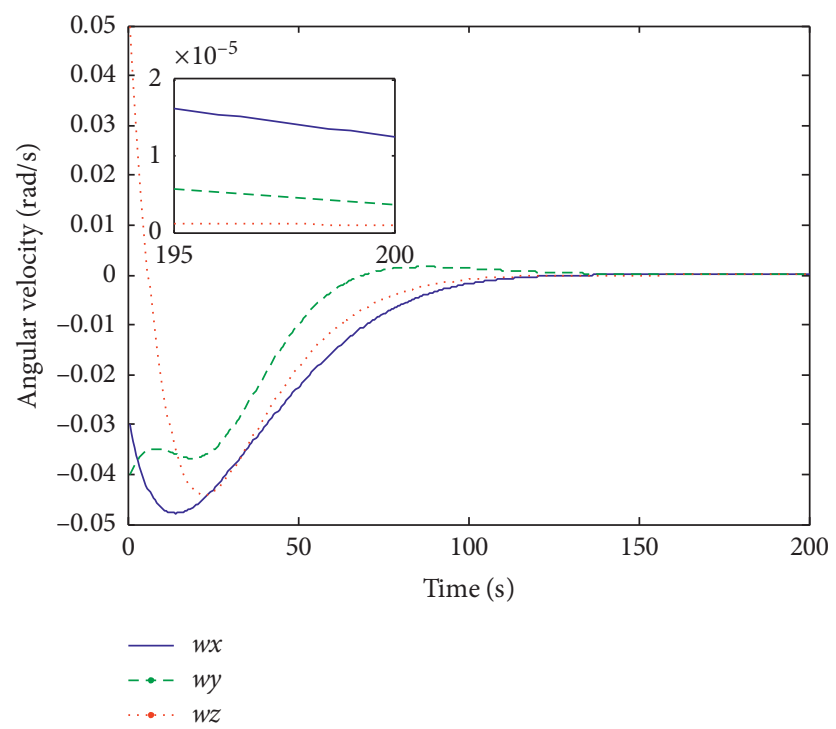

FIGURE 4: Curve of angular velocity.

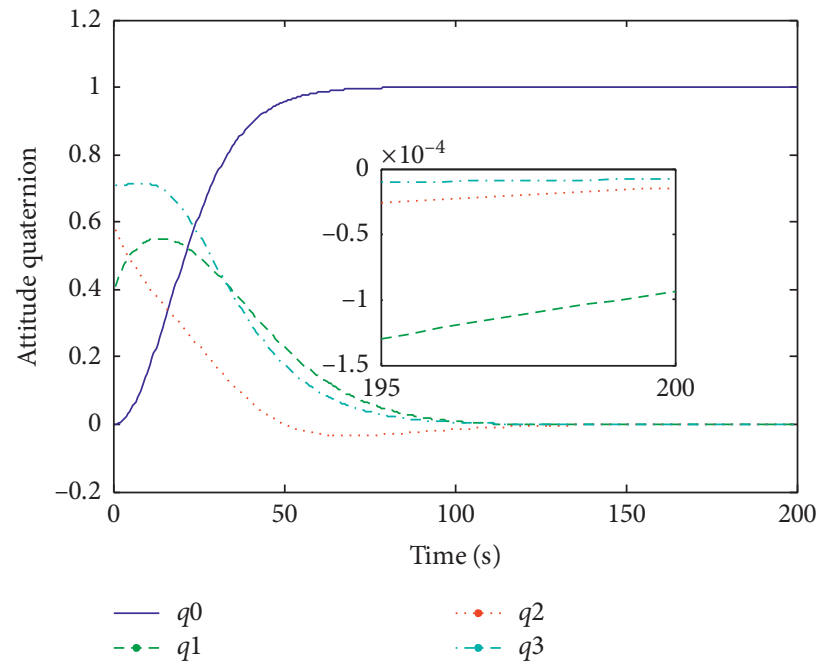

Figure 5: Curve of attitude quaternion.

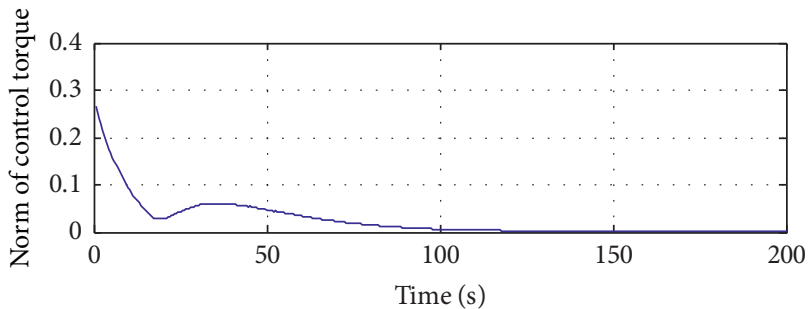

(a)

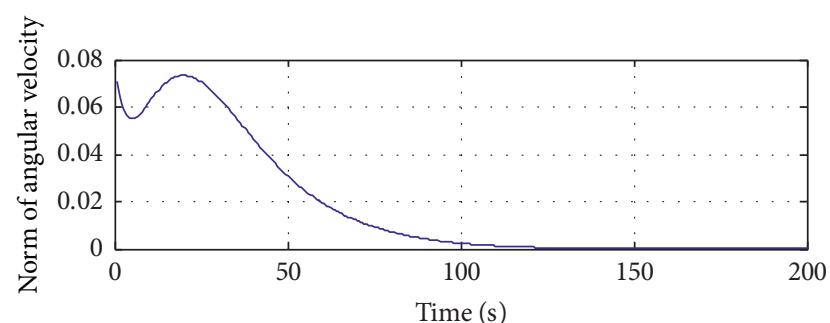

(b)

FIgURE 6: Curve of angular velocity and control torque norm. 
TABLE 1: Simulation results comparison.

\begin{tabular}{lcccc}
\hline & $\begin{array}{c}\text { Convergence } \\
\text { time }(\mathrm{s})\end{array}$ & $\begin{array}{c}\text { Max control } \\
\text { torque }\end{array}$ & $\begin{array}{c}\text { Max angular } \\
\text { velocity }\end{array}$ & Conclusion \\
\hline $\begin{array}{l}\text { Standard sliding mode with } \\
\text { larger parameter }\end{array}$ & 90 & $1.3 \mathrm{Nm}$ & $0.18 \mathrm{rad} / \mathrm{s}$ & $\begin{array}{c}\text { Best convergence rate, but control torque and angular } \\
\text { velocity exceed system upper bound }\end{array}$ \\
$\begin{array}{l}\text { Standard sliding mode with } \\
\text { larger parameter }\end{array}$ & 150 & $0.27 \mathrm{Nm}$ & $0.08 \mathrm{rad} / \mathrm{s}$ & $\begin{array}{c}\text { Torque and velocity constraints are satisfied, but } \\
\text { convergence rate is the slowest }\end{array}$ \\
$\begin{array}{l}\text { Dynamic sliding mode } \\
\text { proposed in this paper }\end{array}$ & 80 & $0.5 \mathrm{Nm}$ & $0.08 \mathrm{rad} / \mathrm{s}$ & $\begin{array}{r}\text { Same convergence rate as Case 1, and torque and } \\
\text { velocity constraints are satisfied }\end{array}$ \\
\hline
\end{tabular}

by selecting the small parameter, but the convergence rate of the standard sliding mode is relatively low.

Case 3. Dynamic sliding mode proposed in this paper.

Based on previous simulation, it could be found that system performance is mainly determined by $k$, and $k_{s}$ determines the initial control torque. Also, based on the update law, it could be found that larger $p$ and $r$ should be selected and the constraints should be satisfied. Hence, the following parameters are selected:

$$
k_{s}=2, k(0)=0.05, p=0.5, r=0.15 \text {. }
$$

Based on equations (27) and (32), it could be found that the constraints on control parameters are all satisfied with equation (36).

The simulation results are shown as follows (Table 1).

Based on Figures 7 and 8, it could be found that system convergence time is about $80 \mathrm{~s}$ which is at the same level with Case 1. Comparing simulation results of Case 3 with that of Case 1 and Case 2, it could be easily found that the sliding mode parameter in Case 3 is the same as Case 2 $(k=0.05)$, and the convergence time in Case 3 is about half of Case 2. And, the sliding mode parameter in Case 3 is $20 \%$ of Case $1(k(0)=0.05$ in Case 3 and $k=0.25$ in Case 1$)$, and the convergence time is at the same level. This demonstrates that, by implementing the update law of the sliding mode parameter, system convergence time could be largely improved.

Based on Figure 9, it could be found that system control torque and angular velocity are lower than the system upper bound during the whole control torque; however, in Case 1, this property does not hold. This demonstrates that the proposed method could improve the system convergence rate, while the demand control torque and angular velocity are relatively small compared with the standard sliding mode. Also, this demonstrates the effectiveness of the constraints on control parameters (27) and (32) proposed in this paper.

Based on Figure 10, it could be found that sliding mode parameter $k$ is norm upper bounded, and the dynamic sliding mode proposed in this paper does not have the singularity issue. Also, it could be found that the basic structure of the standard sliding mode is maintained; hence, the system has strong robustness to model uncertainty and unknown disturbance torque.

Above all, it could be concluded that the proposed method could improve the system convergence rate without

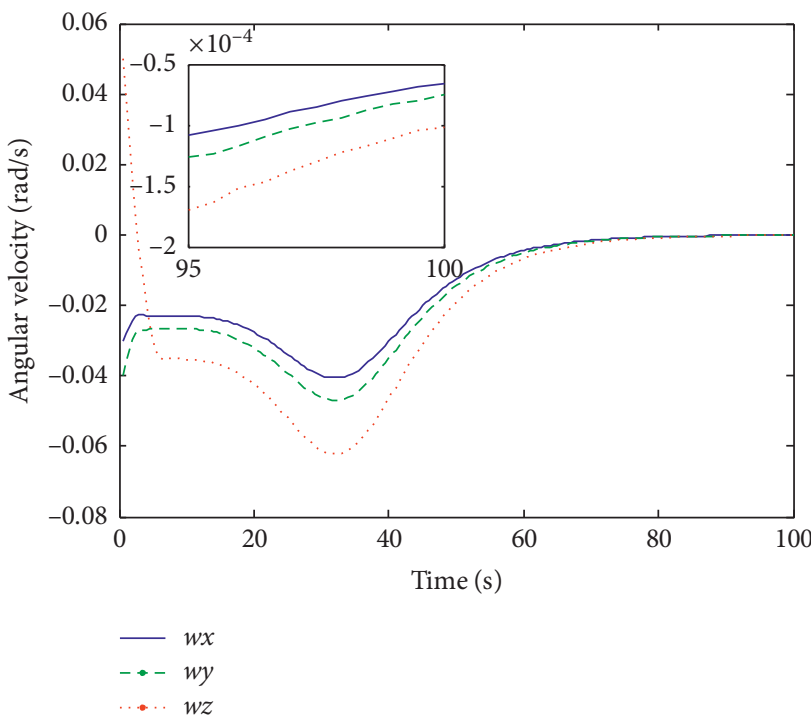

FIgURE 7: Curve of angular velocity.

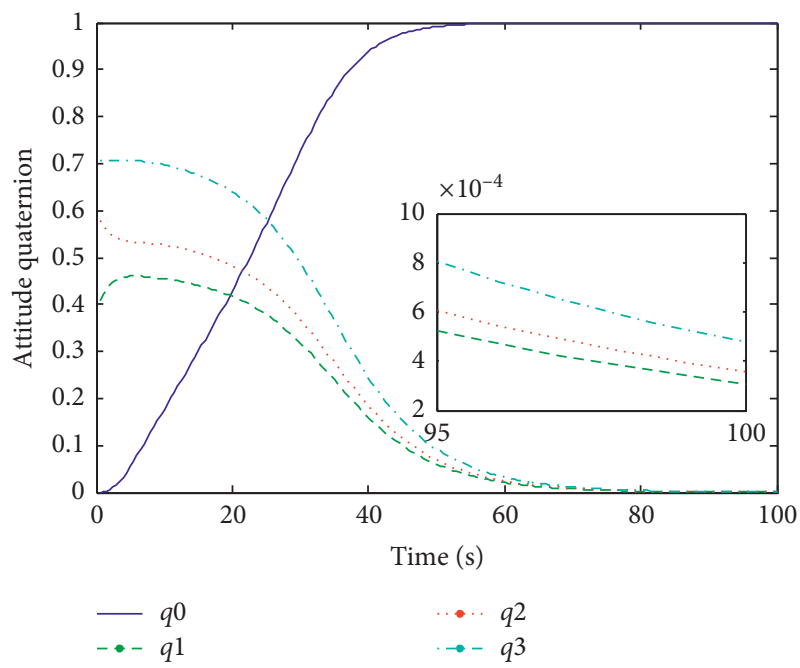

Figure 8: Curve of attitude quaternion.

causing control torque and angular velocity saturation issue. The method proposed in this paper could deal with the low convergence rate issue of the standard sliding mode and maintain its advantages. 


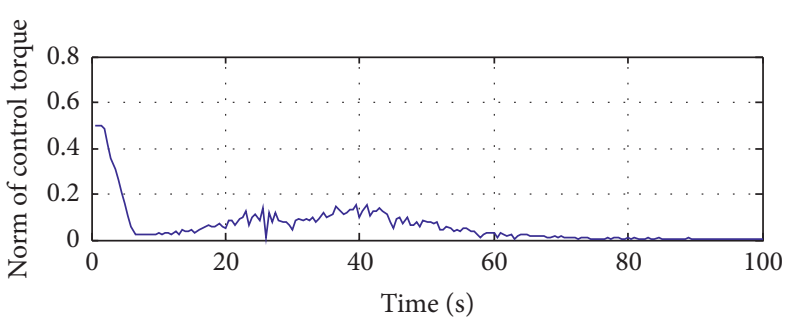

(a)

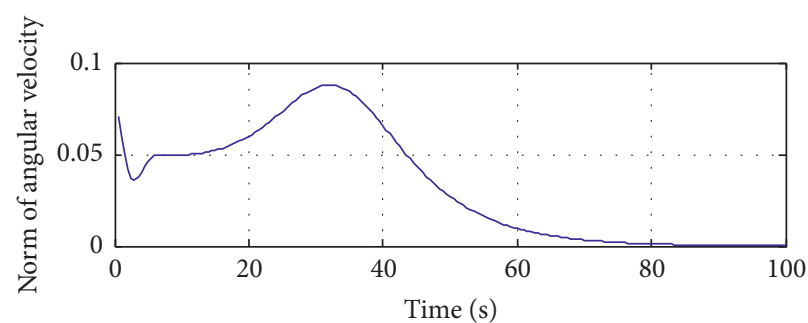

(b)

FIgURE 9: Curve of angular velocity and control torque norm.

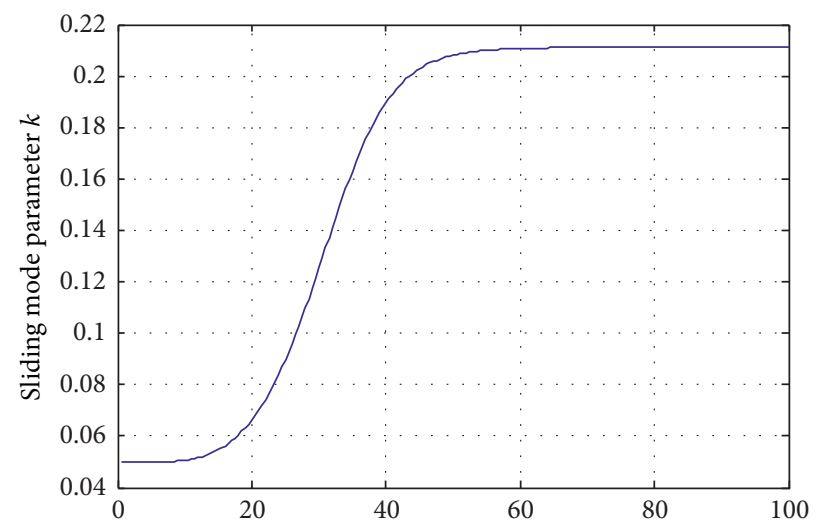

Figure 10: Curve of sliding mode parameter $k$.

\section{Conclusion}

In this paper, a dynamic sliding mode with the time varying sliding mode parameter is proposed for satellite attitude control based on the standard sliding mode. The focus of the proposed method is to deal with the low convergence rate issue and maintain other advantages of the standard sliding mode. It is also proved that the designing of angular velocity trajectory properly is the key to improve convergence rate and system performance. The results of this paper are listed as follows:

(1) The reverse of angular velocity and attitude quaternion is the key to improve the system convergence rate, and the low convergence rate is caused by the descend of angular velocity in the standard sliding mode. By enlarging the norm of angular velocity and maintaining the reverse property, the system convergence rate could be improved.

(2) By implementing the update law of the sliding mode parameter, the system could have better performance compared with the fixed parameter sliding mode. System control torque and velocity are utilized more effectively by the method of variable structure sliding mode. Moreover, by designing the update law properly, the system could both have good convergence rate and strong robustness.

(3) System control torque and angular velocity constraints could be satisfied by selecting control parameters properly, and the method proposed in this paper is suitable for engineering practice.
The dynamic sliding mode method proposed in this paper is enlightening for the sliding mode controller design, and later works could focus on the dynamic sliding mode with finite-time or fixed-time convergence rate.

\section{Data Availability}

The data used to support the findings of this study are available from the corresponding author upon request.

\section{Conflicts of Interest}

The authors declare that they have no conflicts of interest regarding the publication of this paper..

\section{Acknowledgments}

This work was supported partially by National Natural Science Foundation of China (Project no. 61903289). The authors greatly appreciate the above financial support.

\section{References}

[1] B. Xiao and S. Yin, "A structure simple controller for satellite attitude tracking maneuver," IEEE Transactions on Industrial Electronics, vol. 64, no. 2, pp. 1436-1446, 2017.

[2] B. Xiao, S. Yin, and H. J. Gao, "Tracking control of robotic manipulators with uncertain kinematics and dynamics," IEEE Transactions on Industrial Electronics, vol. 63, no. 10, pp. 6439-6449, 2016. 
[3] Q. L. Hu, C. L. Du, L. H. Xie, and Y. Y. Wang, "Discrete-time sliding mode control with time-varying surface for hard disk drives," IEEE Transactions on Control Systems Technology, vol. 17, no. 1, pp. 175-183, 2009.

[4] Q. L. Hu, Y. M. Zhang, X. Huo, and B. Xiao, "Adaptive integral-type sliding mode control for spacecraft attitude maneuvering under actuator stuck failures," Chinese Journal of Aeronautics, vol. 24, no. 1, pp. 32-45, 2011.

[5] S. N. Wu, R. Wang, and Z. G. Wu, "Robust attitude maneuver control of spacecraft with reaction wheel low-speed friction compensation," Aerospace Science and Technology, vol. 43, no. 218, p. 213, 2015.

[6] S. N. Wu, Z. W. Sun, and H. Deng, "Robust sliding mode controller design for globally fast attitude tracking of target spacecraft," International Journal of Innovative Computing Information and Control, vol. 7, no. 5, pp. 2087-2098, 2011.

[7] H. Z. Liang, J. Y. Wang, and Z. W. Sun, "Robust decentralized coordinated attitude control of spacecraft formation," Acta Astronautica, vol. 69, no. 5-6, pp. 280-288, 2011.

[8] H. Z. Liang, Z. W. Sun, and J. Y. Wang, "Finite-time attitude synchronization controllers design for spacecraft formations via behavior-based approach," Journal of Aerospace Engineering, vol. 11, pp. 1737-1753, 2013.

[9] J. Y. Wang, H. Z. Liang, and Z. W. Sun, "Dual-quaternionbased finite-time control for spacecraft tracking in six degrees of freedom," Journal of Aerospace Engineering, vol. 3, pp. 528-545, 2013.

[10] J. Y. Wang, H. Z. Liang, Z. W. Sun, and S. J. Zhang, "Finitetime control for spacecraft formation with dual-numberbased description," Journal of Guidance Control and Dynamics, vol. 35, no. 3, pp. 950-962, 2012.

[11] S. N. Wu, G. Radice, and Z. W. Sun, "Robust finite-time control for flexible spacecraft attitude maneuver," Journal of Aerospace Engineering, vol. 27, no. 1, pp. 185-190, 2014.

[12] Y. Li, D. Ye, and Z. Sun, "Robust finite time control algorithm for satellite attitude control," Aerospace Science and Technology, vol. 68, pp. 46-57, 2017.

[13] D. B. Jovan and M. L. Sai, "Robust tracking control design for spacecraft under control input saturation," Journal of Guidance Control and Dynamics, vol. 27, no. 4, pp. 627-633, 2004.

[14] D. Verbin and V. J. Lappas, "Rapid rotational maneuvering of rigid satellites with hybrid actuators configuration," Journal of Guidance Control and Dynamics, vol. 36, no. 2, pp. 532-547, 2013.

[15] D. Verbin, "Rapid rotational maneuvering of rigid satellites with reaction wheels," ournal of Guidance Control and Dynamics, vol. 36, pp. 1538-1544, 2013.

[16] Y. Li, D. Ye, and Z. Sun, "Time efficient sliding mode controller based on bang-bang logic for satellite attitude control," Aerospace Science and Technology, vol. 75, pp. 342-352, 2018.

[17] Y. Li and D. Ye, "Near time-optimal controller based on analytical trajectory planning algorithm for satellite attitude maneuver," Aerospace Science and Technology, vol. 84, pp. 497-509, 2019.

[18] Y. Li, Z. W. Sun, and D. Ye, "Time efficient robust PID plus controller for satellite attitude stabilization control considering angular velocity and control torque constraint," Journal of Aerospace Engineering, vol. 30, no. 5, 2017.

[19] X. D. Sun, L. Y. Feng, K. Diao, and Z. B. Yang, “An improved direct instantaneous torque control based on adaptive terminal sliding mode for a segmented-rotor SRM," IEEE Transactions on Industrial Electronics, vol. 99, 2020.

[20] X. D. Sun, J. H. Cao, G. Lei, and Y. G. Guo, “A composite sliding mode control for SPMSM drives based on a new hybrid reaching law with disturbance compensation," IEEE Transactions on Industrial Electronics, vol. 99, 2021.

[21] X. D. Sun, J. L. Wu, G. Lei, and Y. G. Guo, "Torque ripple reduction of SRM drive using improved direct torque control with sliding mode controller and observer," IEEE Transactions on Industrial Electronics, vol. 99, 2020. 